\title{
Development of a Novel Hydrostatic Continuously Variable Transmission with Fast Path Switching
}

\author{
Kei Sugihara $^{\mathrm{a}^{*}, \text { Takahiro Nozaki }}{ }^{\mathrm{a}, \mathrm{b}^{*}}$, Toshiyuki Murakami ${ }^{\mathrm{a}}$

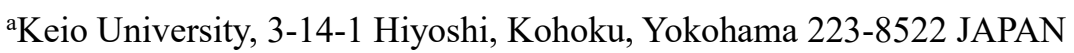 \\ ${ }^{\mathrm{b}}$ Massachusetts Institute of Technology, 77 Massachusetts Avenue, Cambridge, Massachusetts, 02139 USA \\ *Corresponding Author: nozaki@sd.keio.ac.jp
}

\begin{abstract}
Recently, human-robot collaboration is required in many kinds of fields. To develop robotic systems for collaboration with humans, a wide driving range of robotic actuators is necessary. For example, robots for rehabilitation have to conduct some tasks, like supporting human and moving fast. Backdrivability is also necessary for robotic actuators from a viewpoint of safety. A hydraulic closed circuit called an electro-hydrostatic actuator (EHA) is utilized for robotic actuators since they have high power/weight ratio and backdrivability. However, EHA cannot achieve characteristics of both high velocity driving and high force driving since a reduction ratio is constant. This paper proposes a novel hydrostatic continuously variable transmission that has two oil paths with a solenoid valve. The solenoid valve switches between two discrete reduction ratios by switching two oil paths. Furthermore, this paper realizes a continuously variable reduction ratio by switching the two oil paths quickly. This paper confirms that the reduction ratio changes continuously and widely by simulations.
\end{abstract}

Keywords: Continuously variable transmission, hydraulic systems, solenoid valve.

\section{Introduction}

In recent years, cooperation between robots and humans is required not only in industrial fields, but also human assistance such as rehabilitation. Most conventional industrial robots conduct only specific tasks ${ }^{(1)}$. However, robots for rehabilitation fields have to conduct some tasks, like supporting human and moving fast. To develop robotic systems for collaboration with humans, a wide driving range of robotic actuators is necessary. Robotic actuators need to drive in high speed and output high force since required driving characteristics are different among each task. Furthermore, robots are required to move flexibly against unexpected disturbances by humans. In other words, high backdrivability is needed to develop a flexible actuator ${ }^{(2)(3)}$.

Hydraulic actuators, which exhibit large outputs, have been utilized widely in the field of construction. Currently, they have been actively researched for robotic actuators ${ }^{(4)}$. Hydraulic systems have two types, open circuits, and closed circuits. Hydraulic actuators are driven by hydraulic pumps and servo valves in open circuits. A servo valve is an electrically operated valve that controls how hydraulic fluid is sent to a hydraulic actuator. Open circuits can control the desired both flow rate and pressure by using servo valves. In other words, the hydraulic actuators have a wide driving range. However, open-loop hydraulic circuits have no backdrivability since the oil direction is one way and servo valves blocked external forces.

On the other hand, a hydraulic closed circuit called an electro-hydrostatic actuator (EHA) is driven by servo pumps coupling with servo motors ${ }^{(5)}$. EHAs are not needed to put large oil tanks and have backdrivability since oil direction is bidirectional. However, they cannot achieve both high speed driving and high force driving since a reduction ratio determined by a ratio of a displacement between a pump and a motor is constant.

This paper proposes a novel hydrostatic continuously variable transmission (CVT). The proposed CVT has two oil paths with a solenoid valve. The solenoid valve switches between two discrete reduction ratios by switching the two oil paths. Furthermore, the proposed CVT can widely change the reduction ratio continuously by switching the solenoid valve quickly. 


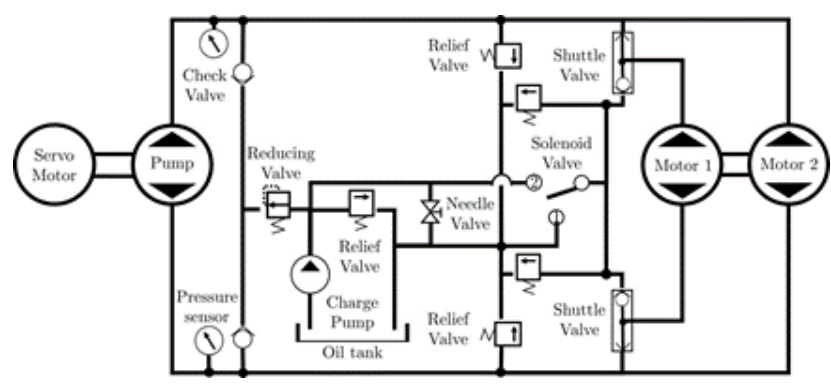

(a) Hydraulic circuit.

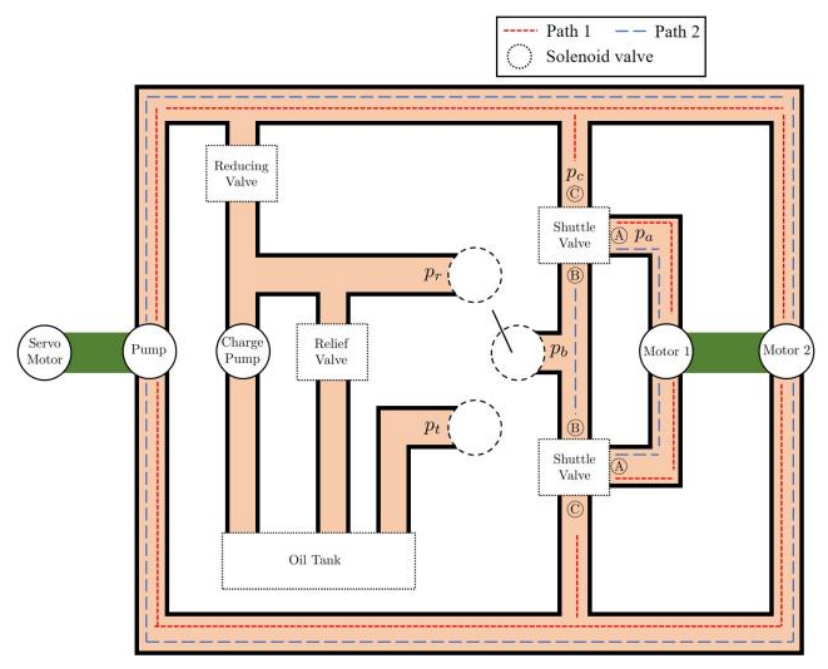

(b) Mechanism.

Fig. 1. Proposed hydrostatic CVT.

This paper is organized as follows. In section 2, the proposed CVT is presented. The control method of a servo motor and a solenoid valve are introduced in section 3 .

Simulations are conducted in section 4. Finally, the conclusions are presented in section 5 .

\section{Proposed Hydrostatic CVT}

\subsection{Hydraulic Circuit}

The proposed hydrostatic CVT is shown in Figures. 1. The proposed CVT is a hydrostatic system similar to EHAs. A servo motor controls a driving of a hydraulic pump and two hydraulic motors are connected by a rotating shaft. A charge pump is connected to keep the minimum pressure of the oil above atmospheric pressure and avoid cavitation. Cavitation is a phenomenon that the air dissolved in the hydraulic cylinder evaporates and forms bubbles when the pressure of the oil drop below the atmospheric pressure in closed hydraulic circuits ${ }^{(7)}$. The proposed hydraulic circuit is protected by installed a charge pump. The charge pressure of

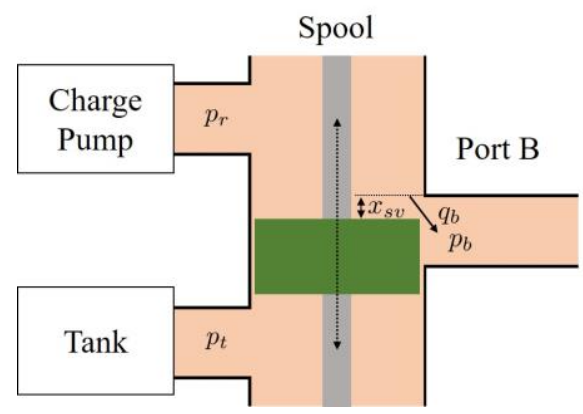

Fig. 2. Flow path of 3-way solenoid valve.

Table 1. Switching patterns of solenoid valve.

\begin{tabular}{|c|c|c|}
\hline Paths & Connect point & Shuttle valve \\
\hline Path 1 & Tank & A to C \\
\hline Path 2 & Charge Pump & B to C \\
\hline
\end{tabular}

the hydraulic circuit is lower than the relief pressure by setting a pressure reducing valve. The relationship among the tank pressure $p_{\mathrm{t}}$, which was assumed to be zero, the charge pressure $\mathrm{p}_{\mathrm{c}}$, and the relief pressure $p_{\mathrm{r}}$ are described as:

$$
p_{\mathrm{t}}<p_{\mathrm{c}}<p_{\mathrm{r}} \text {. }
$$

A solenoid valve and two shuttle valves are used to switch two oil paths. Shuttle valves determine a direction by pressure difference. Figure. 2 shows a flow path of a $3-$ way solenoid valve. The spool moves and opens each path when the voltage applies to the solenoid valve. The spool position $x_{s v}$ is given as

$$
x_{\mathrm{sv}}=k_{\mathrm{vo}} V \text {, }
$$

where $V$ and $k_{\mathrm{vo}}$ represent the input voltage and the spool position gain. Then, the output oil flow $q_{\mathrm{b}}$ and pressure $p_{\mathrm{b}}$ are given as

$$
\begin{gathered}
q_{\mathrm{b}}=k_{\mathrm{sv}} x_{v}\left(s\left(x_{\mathrm{sv}}\right) \sqrt{\frac{2\left|p_{\mathrm{r}}-p_{\mathrm{b}}\right|}{\rho}}+\right. \\
\left.s\left(-x_{\mathrm{sv}}\right) \sqrt{\frac{2\left|p_{\mathrm{b}}-p_{\mathrm{t}}\right|}{\rho}}\right), \\
S\left(x_{\mathrm{v}}\right)=\left\{\begin{array}{l}
1, \text { if } x_{\mathrm{sv}} \leq 0 \\
0, \text { if } x_{\mathrm{sv}}>0
\end{array}\right. \\
s C_{\mathrm{b}} p_{\mathrm{b}}=q_{\mathrm{b}}-K_{\mathrm{l}}\left(p_{\mathrm{b}}-p_{\mathrm{c}}\right),
\end{gathered}
$$

where $q, p, k_{\mathrm{sv}}, \rho, C_{\mathrm{b}}$, and $K_{1}$ represent flow rate, pressure, a flow coefficient, oil density, a compression coefficient, and a leakage coefficient respectively. By applying (3)-(5), the pressure $p_{\mathrm{b}}$ changes by switching the solenoid valve. Switching pattern of the solenoid valve is listed in Table 1 . In the path 1 connected to the tank, the port B pressure becomes lower than the charge pressure. The port $\mathrm{A}$ and the port $\mathrm{C}$ are connected and the port $\mathrm{B}$ is blocked. On the other hand, the pressure of the port B becomes higher than the 


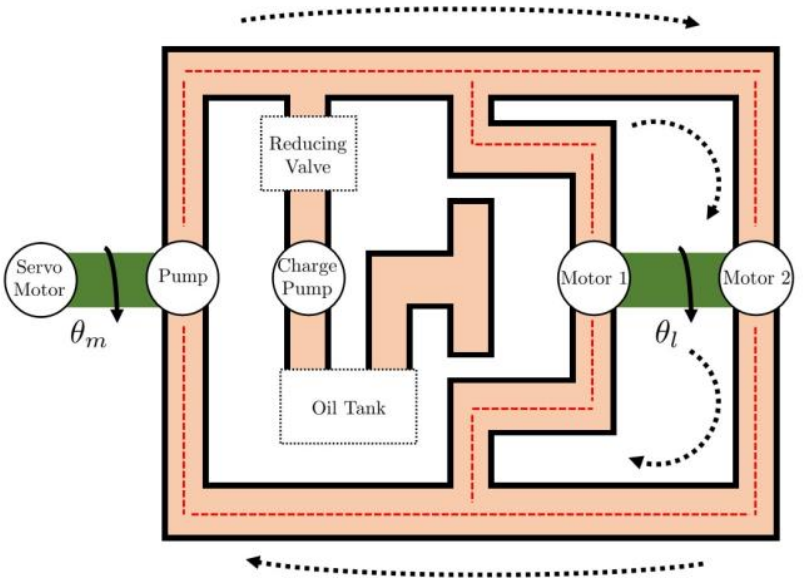

Fig. 3. Path 1.

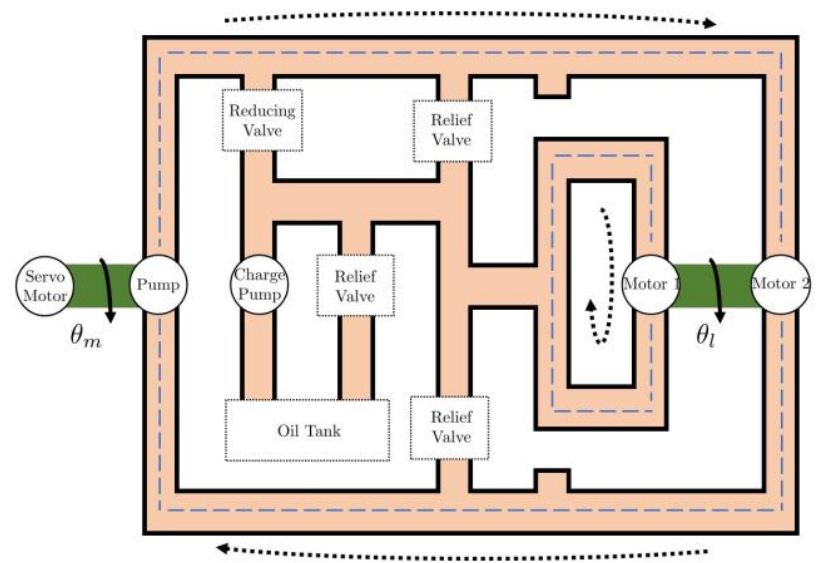

Fig. 4. Path 2.

one of the port $\mathrm{C}$ in the path 2 since the charge pressure is lower than the relief pressure. The port $\mathrm{A}$ and the port $\mathrm{B}$ are connected and the port $\mathrm{C}$ is blocked. The model of the shuttle valve can be described as

$$
p_{\mathrm{a}}=\max \left(p_{\mathrm{b},}, p_{\mathrm{c}}\right) \text {. }
$$

\subsection{Modelling}

Figure. 3 shows the path 1 in the proposed CVT. The red line is the flow path. The motor 1 and the motor 2 rotate by the input pump. The path 2 is shown in figure. 4 . The blue line is the flow path. In the path 2 , the only motor 2 rotates by input pump. Oil flow displaced by the motor 1 flows the loop. The displacement of the hydraulic motor in the path 2 is different from the one of the path 1 . Therefore, the reduction ratio can be switched between two discrete value.

The dynamics of the motor side is given as

$$
J_{\mathrm{m}} \ddot{\theta}_{\mathrm{m}}+B_{\mathrm{m}} \dot{\theta}_{\mathrm{m}}=\tau_{\mathrm{m}}^{\mathrm{ref}}-\frac{D_{\mathrm{m}}}{2 \pi} \Delta \mathrm{p}-\tau_{\mathrm{m}}^{\mathrm{dis}}
$$

where $J, B, D, \theta, \tau$, and $\Delta \mathrm{p}$ represent inertia, viscous coefficient, displacement, angle, torque, and pressure

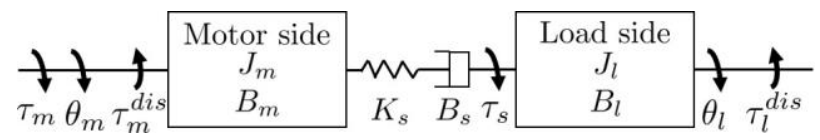

Fig. 5. Two-inertia resonant system.

difference between the input and the output ports respectively. The subscript $m$ stands for an index for the motor side. The superscript ref and dis stand for an index for the reference value and the disturbance value respectively.

The dynamics of the load side is given as,

$$
\begin{aligned}
J_{1} \ddot{\theta}_{1}+B_{1} \dot{\theta}_{1} & =\frac{D_{l}}{2 \pi} \Delta \mathrm{p}-\tau_{\mathrm{l}}^{\mathrm{dis}}, \\
D_{1} & =\left(D_{11}+D_{12}, D_{12}\right),
\end{aligned}
$$

where $D_{11}$ and $D_{12}$ represent the displacement of the motor 1 and the motor 2 respectively. The subscript 1 represents an index for the load side. The pressure difference $\Delta p$ is expressed by the oil compression as follows:

$$
\begin{aligned}
s \Delta p & =\frac{1}{C_{b}}\left(q_{\mathrm{m}}-q_{1}-K_{\mathrm{l}} \Delta p\right), \\
& =\frac{1}{C_{\mathrm{b}}}\left(\frac{D_{\mathrm{m}}}{2 \pi} \dot{\theta}_{\mathrm{m}}-\frac{D_{\mathrm{l}}}{2 \pi} \dot{\theta}_{\mathrm{l}}-k_{\mathrm{l}} \Delta p\right), \\
& =\frac{D_{\mathrm{m}}}{2 \pi C_{\mathrm{b}}}\left(\dot{\theta}_{\mathrm{m}}-\frac{D_{\mathrm{l}}}{D_{\mathrm{m}}} \dot{\theta}_{\mathrm{l}}-\frac{2 \pi}{D_{\mathrm{m}}} \Delta p\right) .
\end{aligned}
$$

Finally, the dynamic of the torsional torque $\tau_{\mathrm{s}}$ is given as

$$
\begin{aligned}
& \tau_{\mathrm{s}}=K_{\mathrm{s}}\left(\theta_{\mathrm{m}}-R \theta_{\mathrm{l}}-\frac{1}{B_{\mathrm{s}}} \int \tau_{\mathrm{s}}\right), \\
& K_{\mathrm{s}}=\frac{1}{C_{b}}\left(\frac{D_{\mathrm{m}}}{2 \pi}\right)^{2}, \\
& B_{\mathrm{s}}=\frac{1}{K_{l}}\left(\frac{D_{\mathrm{m}}}{2 \pi}\right)^{2}, \\
& R=\left(\frac{D_{\mathrm{l} 1}+D_{\mathrm{l} 2}}{D_{\mathrm{m}}}, \frac{D_{\mathrm{l} 2}}{D_{\mathrm{m}}}\right),
\end{aligned}
$$

where $K_{\mathrm{s}}, B_{\mathrm{s}}$, and $R$ represent a spring coefficient of oil, a viscous coefficient, and reduction ratios respectively. Modeling and parameters except the reduction ratios are the same between the path 1 and the path 2. By applying (7)-(14), the proposed CVT is modeled as two-inertia resonant systems as shown in Figure. 5.

\section{Control}

\subsection{Position Control}

The motor-side angle is controlled by the servo motor. The block diagram of the position control is shown in Figure. 6 . $K_{\mathrm{p}}$ and $K_{\mathrm{v}}$ represent feedback gains of position and velocity for a position controller. The superscripts $\mathrm{cmd}$ stands for 


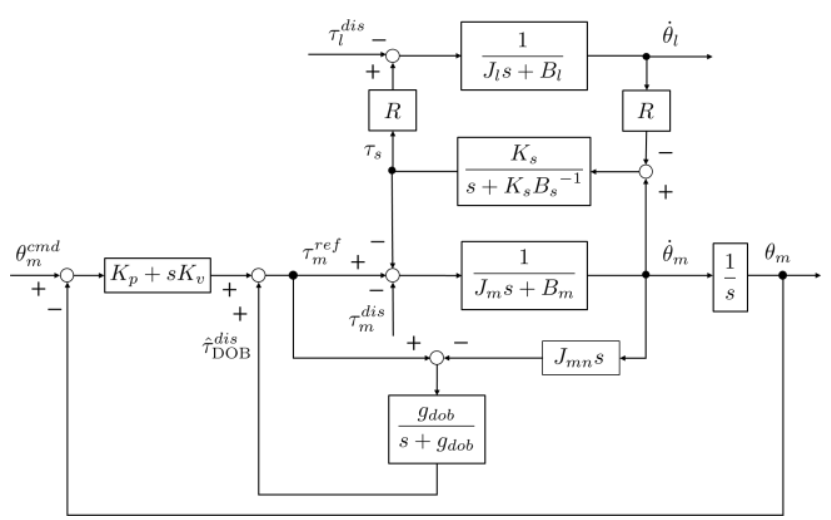

Fig. 6. Block diagram of position control.

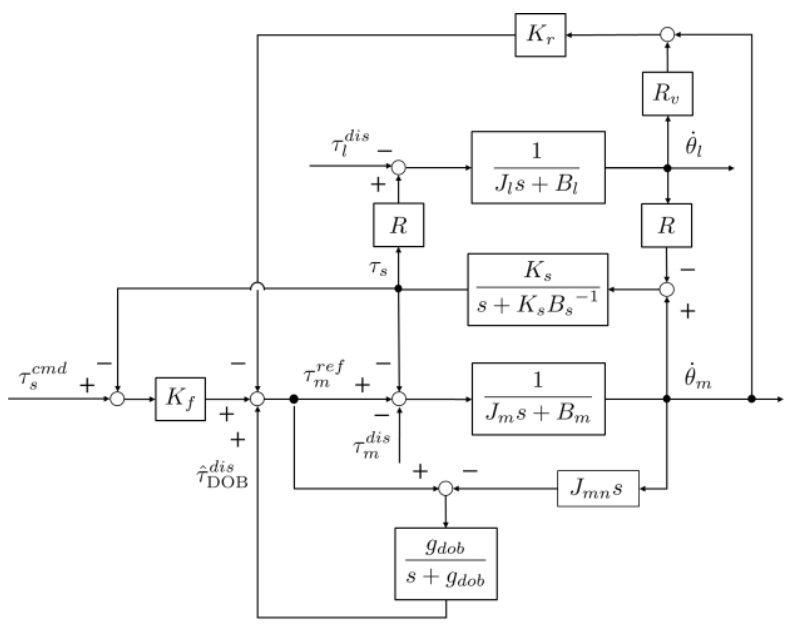

Fig. 7. Block diagram of torsional torque control.

command values and response values respectively. Disturbance observer (DOB) was implemented to achieve robust control $^{(8)}$. The estimated disturbance value $\hat{\tau}_{\text {dob }}^{\text {dis }}$ is described as follows:

$$
\begin{aligned}
\hat{\tau}_{\mathrm{dob}}^{\mathrm{dis}} & =\hat{\tau}_{\mathrm{m}}^{\mathrm{dis}}+\hat{\tau}_{\mathrm{s}}, \\
& =\frac{g_{\mathrm{dob}}}{s+g_{\mathrm{dob}}}\left(\tau_{\mathrm{m}}^{\mathrm{ref}}-J_{\mathrm{mn}} \ddot{\theta}_{\mathrm{m}}\right),
\end{aligned}
$$

where $g_{\text {dob }}$ is the cut-off frequency of the low-pass filter. $\widehat{\bigcirc}$ stands for estimated values. As $g_{\text {dob }}$ sets higher, the control system becomes robust against the disturbance.

\subsection{Torsional Torque Control}

The block diagram of the torsional torque control is shown in Figure. 7. Torsional torque is controlled with a torque sensor. $K_{\mathrm{f}}$ is a feedback gain for a force controller. $K_{\mathrm{r}}$ is a feedback gain to suppress two-inertia resonant systems ${ }^{(9)}$. Then, the input torque reference is given as

$$
\begin{aligned}
\tau_{\mathrm{m}}^{\mathrm{ref}}= & K_{\mathrm{f}}\left(\tau_{\mathrm{s}}^{\mathrm{cmd}}-\tau_{\mathrm{s}}\right)+\tau_{\mathrm{dob}}^{\mathrm{dis}}+ \\
& K_{\mathrm{r}}\left(\omega_{\mathrm{m}}-R_{\mathrm{v}} \omega_{\mathrm{l}}\right),
\end{aligned}
$$

where $R_{\mathrm{v}}$ represents the desired variable reduction ratio.

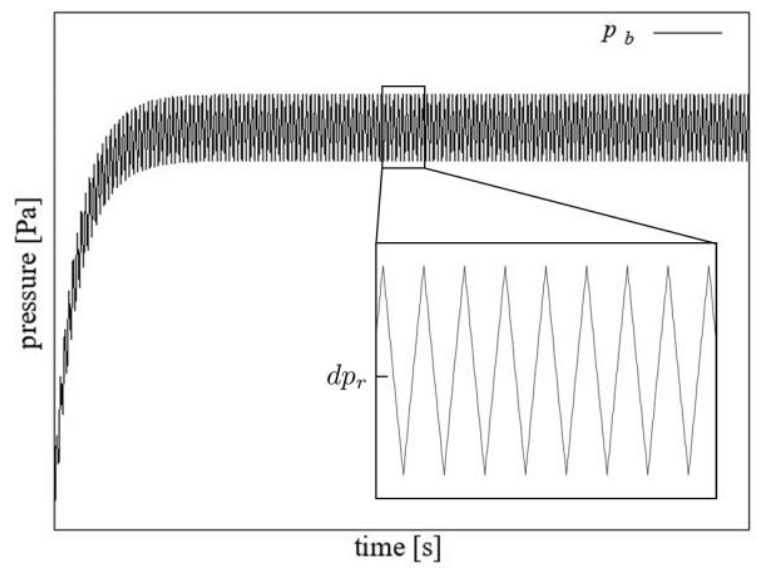

Fig. 8. Port B pressure when $d=0.5$.

\subsection{Reduction Ratio Control}

The continuously variable reduction ratio is achieved by switching the solenoid valve quickly. The solenoid valve is controlled by a pulse width modulation (PWM) signal with adjustable duty ratio and frequency ${ }^{(10)(11)}$. Input voltage is described as

$$
\begin{aligned}
V & =s(t) V_{0}, \\
S\left(x_{\mathrm{v}}\right) & =\left\{\begin{array}{l}
1, \quad \text { if } 0<t \leq d T \\
-1, \text { if } d T<t \leq T
\end{array},\right.
\end{aligned}
$$

where $V_{0}, d$, and $T$ represent the initial voltage, PWM signal duty ratio, and PWM signal period. By applying (3)-(5) and (18)-(19), the port B pressure in PWM control is shown in Figure 8. As shown in Figure 8, the port B pressure is oscillating. When switching frequency is sufficiently fast and the duty ratio sets 0.5 , the port B pressure in the steady state $\bar{p}_{\mathrm{b}}$ is described as

$$
\overline{p_{\mathrm{b}}}=\frac{p_{\mathrm{r}}}{2} \text {. }
$$

Then, the charge pressure is set as follows:

$$
p_{\mathrm{c}}=\overline{p_{\mathrm{b}}},
$$

By setting (21), the port B pressure is oscillating around the charge pressure and two oil paths are switched quickly. The average value of reduction ratio $R_{0.5}$ is given as

$$
R_{0.5}=\frac{R_{1}+R_{2}}{2} .
$$

Control of the input voltage is required to make the average value of the reduction ratio consistent with the desired variable reduction ratio. The relative angular velocity $W_{\mathrm{v}}$ is given as

$$
W_{\mathrm{v}}=\omega_{\mathrm{m}}-R_{\mathrm{v}} \omega_{\mathrm{l}} \text {. }
$$

$W_{\mathrm{v}}$ should be zero when the average reduction ratio is same with the desired one. In the position control, the average reduction ratio of the proposed CVT has to be decreased 
Table 2. Parameters of simulations.

\begin{tabular}{|c|c|c|c|}
\hline Parameters & Values & Parameters & Values \\
\hline$J_{\mathrm{m}}$ & $\begin{array}{c}0.00063 \\
\mathrm{~kg} \cdot \mathrm{m}^{2}\end{array}$ & $k_{\mathrm{vo}}$ & $\begin{array}{c}0.075 \\
\mathrm{~mm} / \mathrm{V}\end{array}$ \\
\hline$J_{1}$ & $0.5 \mathrm{~kg} \cdot \mathrm{m}^{2}$ & $k_{\mathrm{sv}}$ & $1.07 \mathrm{E}-5$ \\
\hline$B_{\mathrm{m}}$ & $\begin{array}{c}0.0003 \\
\mathrm{Nms} / \mathrm{rad}\end{array}$ & $V_{0}$ & $10.0 \mathrm{~V}$ \\
\hline$B_{1}$ & $5.0 \mathrm{Nms} / \mathrm{rad}$ & $g_{\text {dob }}$ & $150 \mathrm{rad} / \mathrm{s}$ \\
\hline$B_{\mathrm{s}}$ & 0.0915 & $K_{\mathrm{p}}$ & 6400 \\
\hline$K_{\mathrm{s}}$ & $\begin{array}{c}\mathrm{Nms} / \mathrm{rad} \\
\mathrm{Nm} / \mathrm{rad}\end{array}$ & $K_{\mathrm{v}}$ & 160 \\
\hline$R_{1}$ & 100 & $K_{\mathrm{f}}$ & 100 \\
\hline$R_{2}$ & 20 & $K_{\mathrm{r}}$ & 0.01 \\
\hline$\rho$ & $900 \mathrm{~kg} / \mathrm{m}^{-3}$ & & \\
\hline \multicolumn{5}{|r}{} \\
\hline
\end{tabular}

when the relative angular velocity is a positive value. It means that the time opening the path 1 in a switching period needs to decrease and the time opening the path 2 in a switching period needs to increase. On the other hand, the average reduction ratio of the proposed CVT has to be increased when the relative angular velocity is a negative value. It means that the time opening the path 1 in a switching period needs to increase and the time opening the path 2 in a switching period needs to decrease. When the positive voltage value is higher than the negative one in the PWM control, the time opening the path 1 becomes long and the time opening the path 2 becomes short. Therefore, the input voltage is controlled as follows :

$$
V=s(t) V_{0}+\frac{K_{\mathrm{iv}}}{s} W_{\mathrm{v}},
$$

where $K_{\text {iv }}$ represent the integral gain. To track the desired reduction ratio in a short time, the voltage gains set higher as the desired reduction ratio becomes large.

In the torsional torque control, the mechanism to control the desired reduction ratio is the same. The relative torque $W_{\mathrm{f}}$ is given as

$$
W_{\mathrm{f}}=R_{v} \tau_{\mathrm{s}}-\tau_{\mathrm{l}}^{\mathrm{dis}}
$$

$W_{\mathrm{f}}$ should be zero when the average reduction ratio is same with the desired one. The average reduction ratio of the proposed CVT has to be increased when the relative angular torque is a positive value. average reduction ratio of the proposed CVT has to be decreased when the relative angular velocity is a negative value. Then, the input voltage is controlled as follows:

$$
V=s(t) V_{0}-\frac{K_{\text {if }}}{s} W_{\mathrm{f}},
$$

where $K_{\text {if }}$ represents the integral gain. To track the desired reduction ratio in a short time, the voltage gains set higher as the desired reduction ratio is small.

\section{Simulation}

\subsection{Condition}

Simulations were conducted to confirm the continuously variable reduction ratio of the proposed CVT. Parameters for simulations are listed in Table 2. By assuming the switching period of the solenoid valves was $60 \mathrm{~ms}$, the duty ratio was set as 0.5 . The variable reduction ratio was set as 40,60 , and 80 . A servo motor was actuated after time 1.0 in the simulations.

\subsection{Results}

Figures. 9 show responses of the motor-side angle. $\theta_{\mathrm{m}}^{\mathrm{cmd}}$ was set as,

$$
\theta_{\mathrm{m}}^{\mathrm{cmd}}=1.0 t,
$$

where $t$ represents time. As shown in Figure. 9 (a), the response of the load-side angular velocity changed by switching the two oil paths quickly. Figures. 9 (b)-(d) show the enlarged view of Figure. 9 (a) for $40 \mathrm{~ms}$ when $R_{\mathrm{v}}$ was set as 40, 60, and 80. As shown in Figures. 9 (b)-(d), the angular velocity responses were oscillating as the two oil paths were switched.

The load-side disturbance torque responses in the torsional torque control are shown in Figures. 10. The command value of the torsional torque was set as $1 \mathrm{Nm}$ with a ramp-shaped trajectory in this simulation. As shown in Figure. 10 (a), the response of the load- side disturbance torque changed by switching the two oil paths quickly. Figures. 10 (b)-(d) show the enlarged view of Figure. 10 (a) for $40 \mathrm{~ms}$ when $R_{\mathrm{v}}$ was set as 40,60 , and 80. As shown in Figures. 10 (b)- (d), the torsional torque responses were oscillating as the two oil paths were switched.

The comparisons of the reduction ratios between the simulation value and the theoretical one are listed in Table 3. The simulation value is calculated by the load-side angular velocity and the torsional torque from 4 seconds to 5 seconds. From Table 3, the simulated reduction ratios corresponded with the theoretical one. As result of the simulations, the reduction ratio could change continuously in a wide range by switching the solenoid valve quickly and changing the input voltage of the solenoid valve. 


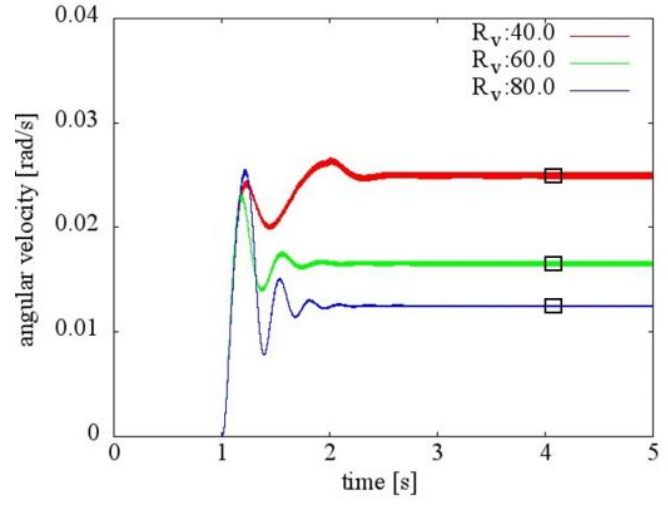

(a) Load-side angular velocity

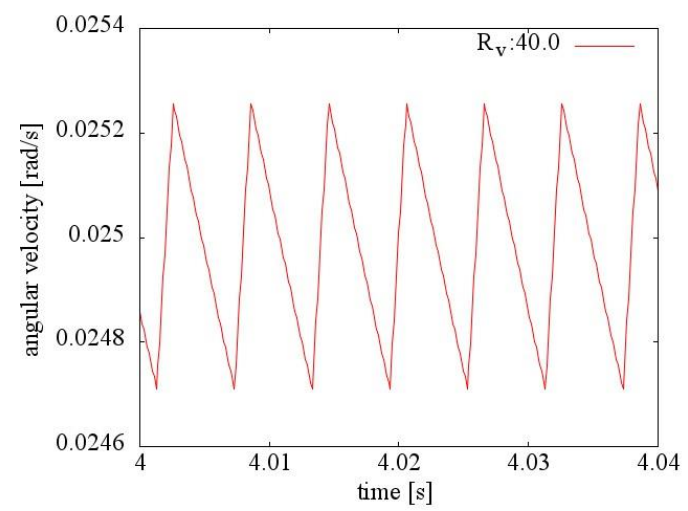

(b) $R_{\mathrm{v}}=40.0$.

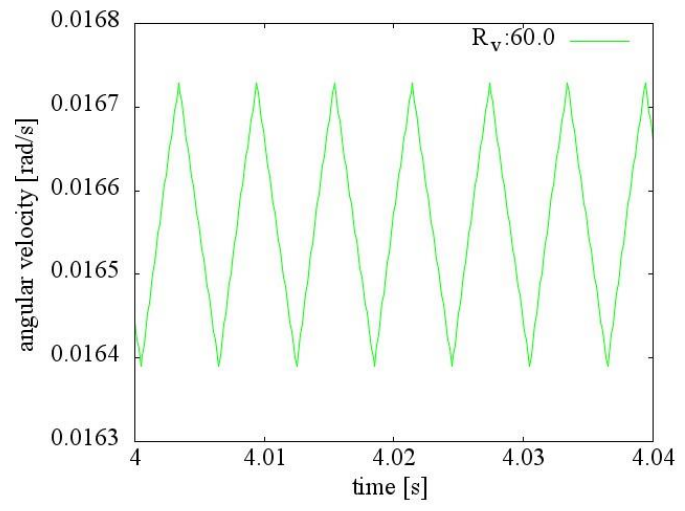

(c) $R_{\mathrm{v}}=60.0$.

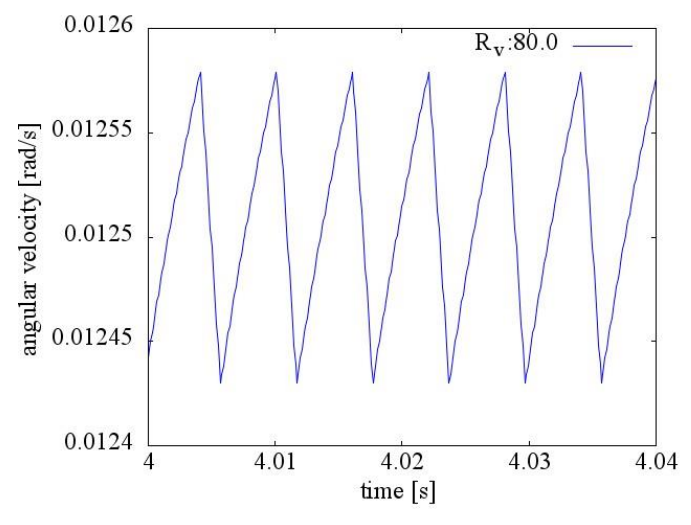

(d) $R_{\mathrm{v}}=80.0$.

Fig. 9. Responses of load-side angular velocity.

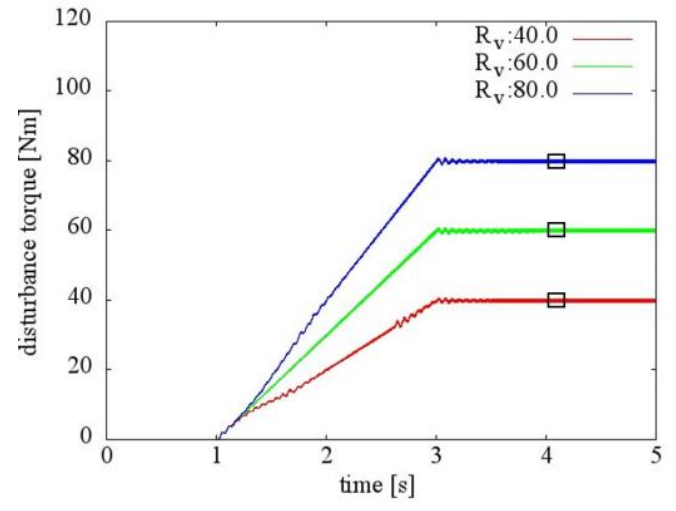

(a) Load-side disturbance torque.

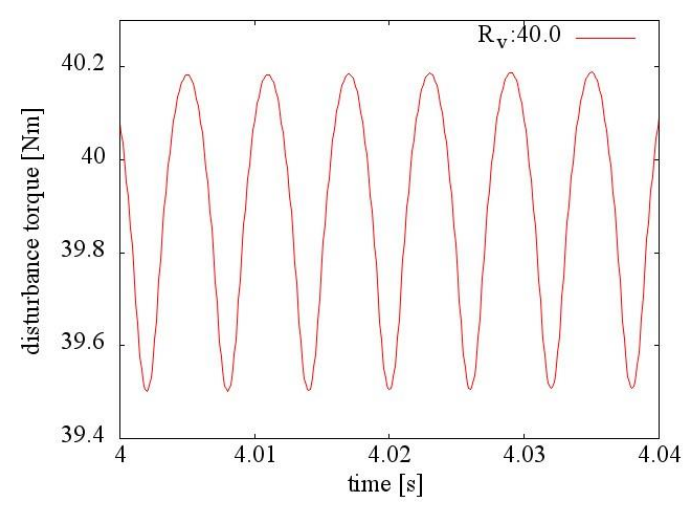

(b) $R_{\mathrm{v}}=40.0$.

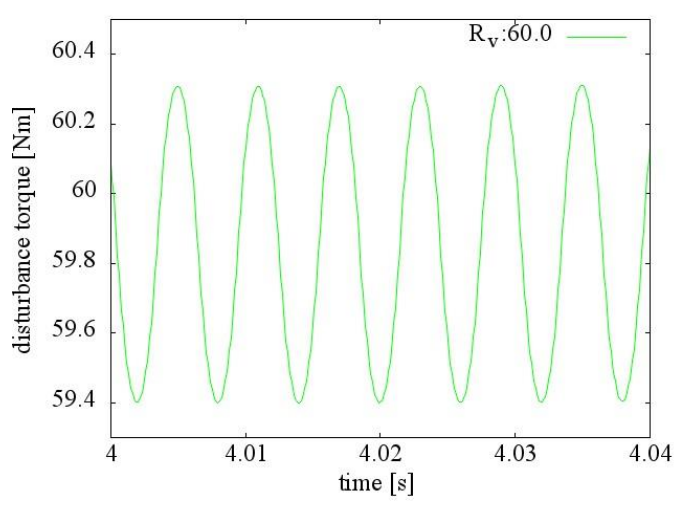

(c) $R_{\mathrm{v}}=60.0$.

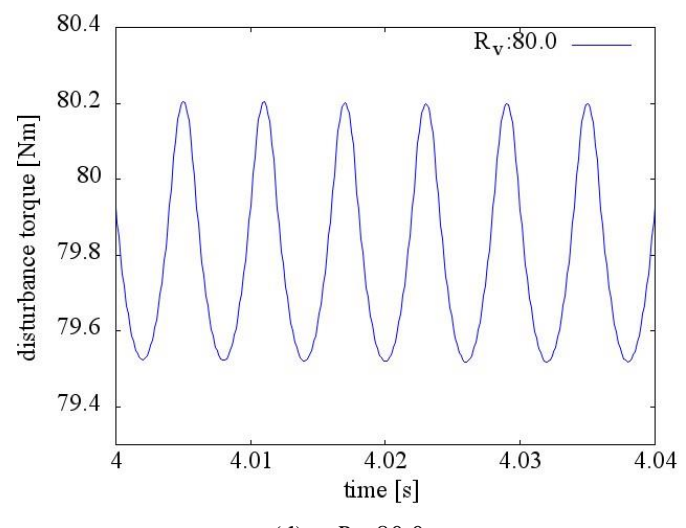

(d) $R_{\mathrm{v}}=80.0$.

Fig. 10. Responses of load-side disturbance. 
Table 3. Comparisons of reduction ratios.

\begin{tabular}{|c|c|c|}
\hline Theoretical value & Figures. 9 & Figures. 10 \\
\hline 40.0 & 40.0 & 39.9 \\
\hline 60.0 & 60.4 & 59.9 \\
\hline 80.0 & 80.0 & 79.8 \\
\hline
\end{tabular}

\section{Conclusions}

In this paper, the novel hydrostatic CVT was proposed. The solenoid valve of the proposed CVT switched between the two discrete reduction ratios. Furthermore, this paper realized that the reduction ratio changed to the desired value by switching the two oil paths quickly and controlling the input voltage of the solenoid valves. This proposed method was confirmed by the simulations. The wide range of variable reduction ratios is achieved by the proposed hydrostatic CVT.

Actuators with CVT can switch between the high speed driving and the high force driving. In other words, a driving range of actuators can expand. Moreover, the proposed mechanism had higher backdrivability than the conventional hydraulic actuators of open circuits.

Future work includes to validate the continuously variable reduction ratio in experiments with comparing the result of the simulation. Hydrostatic systems included nonlinearities. Control scheme compensating these effects had to be developed to achieve the desired reduction ratio.

\section{Acknowledgment}

This work was supported by JSPS KAKENHI Grant Numbers JP20H02135 and JP19KK0367.

\section{References}

(1) S. Thrun, "Toward a framework for human-Robot interaction", Human Computer Interaction, vol. 19, no. 1-2, pp. 9-24, June 2004.

(2) K. Suzumori, "Backdrivability of robots and actuators", Journal of the Robotics Society of Japan, vol. 31, no. 6, pp. 548-551, July, 2013.

(3) N. Tobias and L. Peter, "Improving backdrivability in geared rehabilitation robots", Medical \& biological engineering \& computing, vol. 47, no. 4, pp. 441-447, Feb. 2009.
(4) J. Mattila, J. Koivumaki, D. G. Caldwell, and C. Semini, "A Survey on control of hydraulic robotic manipulators with projection to future trends", IEEE/ASME Transactions on Mechatronics, vol. 22, no. 2, pp. 669680, Apr. 2017.

(5) K. Umeda S. Sakaino, K. Tsuda, T. Sakuma, and T. Tsuji, "Resonance- suppression control for electro-hydrostatic actuator as two-inertia system", IEEJ Journal of Industry Applications, vol. 6, no. 5, pp. 320-327, Sept. 2017.

(6) T. Sakuma S. Sakaino, and T. Tsuji, “A Control Strategy for Electro-hydrostatic Actuator Considering Static Friction, Resonance, and Oil Leakage", IEEJ Journal of Industry Applications, vol. 8, no. 2, pp. 279-286, June 2019.

(7) Y. Nakashima H. Kaminaga, J. Ono, and Y. Nakamura, "Development of backdrivable hydraulic joint mechanism for knee joint of humanoid robots", In 2009 IEEE International Conference on Robotics and Automation, pp. 1577-1582, May 2009.

(8) K. Ohnishi, M. Shibata, and T. Murakami, "Motion control for advanced mechatronics", IEEE/ASME Transactions on Mechatronics, vol. 1, no. 1, pp. 56-67, Mar. 1996.

(9) S. Yamada and H. Fujimoto, "Proposal of high backdrivable control using load-side encoder and backlash", In IECON 2016 - 42nd Annual Conference of the IEEE Industrial Electronics Society, pp. 6429-6434, Oct. 2016.

(10) F. Wang, L. Gu, and Y. Chen, “A continuously variable hydraulic pressure converter based on high-speed on-off valves", Mechatronics, vol. 21, no. 8, pp. 1298-1308, Dec. 2011

(11)F. Wang, L. Gu, and Y. Chen, "A hydraulic pressureboost system based on high-speed on/off valves", IEEE/ASME Transactions on Mechatronics, vol. 18, no. 2, pp. 733-743, Feb. 2013. 